Pacific Journal of Mathematic 


\section{ASYMPTOTIC ESTIMATES FOR LIMIT POINT PROBLEMS}

\section{A. Swanson}

Introduction. The variation of characteristic values and functions of the differential operator $L$ defined by

$$
L x=\frac{1}{k(s)}\left\{-\frac{d}{d s}\left[p(s) \frac{d x}{d s}\right]+q(s) x\right\}
$$

will be studied when the domain of $L$ varies because of a change of boundary conditions. The basic interval is an open interval $\omega_{-}<s<\omega_{+}$ on which $k$ is positive and piecewise continuous, $p$ is positive and differentiable, and $q$ is real-valued and piecewise continuous. For a closed subinterval $[a, b]$ of the basic interval, our purpose is to obtain estimates for the characteristic values $\mu_{a b}$ and characteristic functions $y_{a b}$ of regular Sturm-Liouville problems on $[a, b]$ when $a, b$ are near $\omega_{-}, \omega_{+}$. Such results have been obtained by the author [6] in the case that both $\omega_{-}$and $\omega_{+}$are limit circle singularities in $\mathrm{H}$. Weyl's classification [2, p. 225]. Here the analogous results will be derived in the limit point case and the mixed case (one singularity of each type). To avoid repetition of the preliminary material in [6], we shall usually adhere to the notation and numbering system of [6] without further comment.

6. Basic problems in the limit point and mixed cases. As in $\S 2$, the limits of $\mu_{a b}$ as $a \rightarrow \omega_{-}, b \rightarrow \omega_{+}$are supposed to exist, and accordingly we shall assume that characteristic values $\lambda$ of suitable singular Sturm-Liouville problems for $L$ on $\left(\omega_{-}, \omega_{+}\right)$exist. These singular problems are described as follows when both $\omega_{-}, \omega_{+}$are limit point singularities [4].

Let $L_{0}$ be the differential operator $L-l_{0}, \operatorname{Im} l_{0} \neq 0$. According to a theorem of Weyl [4, p. 45] there exist linearly independent solutions $\varphi_{-}, \varphi_{+}$of $L_{0} \varphi=0$ such that

$$
\varphi_{+} \in \mathfrak{F}_{\omega_{\omega_{+}}}, \quad \varphi_{-} \in \mathfrak{F}_{\omega_{-} \omega}, \quad\left[\varphi_{+} \bar{\varphi}_{-}\right](s)=1
$$

for any $\omega$ satisfying $\omega_{-}<\omega<\omega_{+}$. These solutions are uniquely determined from the normalization condition $\left[\varphi_{+} \varphi_{+}\right]\left(s_{0}\right)=i$ at some point $s_{0}$, to remain fixed in the sequel. (Compare (6.1) with the choice (2.1) of $\varphi_{-}, \varphi_{+}$in the limit circle case.) Let $\mathfrak{D}^{0}$ be the set of all $x$ in the basic Hilbert space $\mathfrak{S}$ (described in $\S 1$ ) which have the following properties: (a) $x$ is differentiable on $\left(\omega_{-}, \omega_{+}\right)$and $x^{\prime}$ is absolutely

Received August 1961. This research was supported by the United States Air Force through the Air Force Office of Scientific Research, under contract number AFOSR 61-89. 
continuous on every closed subinterval of this interval; and (b) $L x \in \mathfrak{S}$. The basic characteristic value problem in the limit point case is then

$$
L x=\lambda x, \quad x \in \mathfrak{D}^{0} .
$$

In this case, $x$ is not restricted by any boundary conditions at $\omega$ and $\omega_{+}$.

Our main assumption is that there exists at least one characteristic value $\lambda$ of this problem. It will be supposed that a corresponding characteristic function $x$ has been selected with $\|x\|=1$.

In the limit circle case, no special assumptions on $L$ at $\omega_{-}$and $\omega_{+}$had to be imposed, but the generality of the boundary operators $U_{a}$ and $U_{b}$ [See (1.5), (2.4)] had to be sacrificed in order to ensure that $\mu_{a b} \rightarrow \lambda$ as $[a, b] \rightarrow\left(\omega_{-}, \omega_{+}\right)$. In the limit point case herein under consideration, the situation is quite different. Some additional restrictions on $L$ as $s \rightarrow \omega_{ \pm}$are clearly. needed to get a point spectrum at all, but then very general boundary operators $U_{a}, U_{b}$ will permit convergence of $\mu_{a b}$ to $\lambda$. The following notation will be used: ${ }^{1}$

$$
\begin{aligned}
\sigma_{a}=\varphi_{-}(a) / \varphi_{+}(a) ; & \sigma_{b}=\varphi_{+}(b) / \varphi_{-}(b) ; \\
\xi_{a}=\left[x(a) / \varphi_{+}(a)\right]\left\|\varphi_{+}\right\|_{a} ; & \xi_{b}=\left[x(b) / \varphi_{-}(b)\right]\left\|\varphi_{-}\right\|^{b} ; \\
\xi_{a}^{*}=\sigma_{a}\left\|\varphi_{+}\right\|_{a} ; & \xi_{b}^{*}=\sigma_{b}\left\|\varphi_{-}\right\|^{b} ; \\
\eta_{a}=U_{a} \varphi_{-} / U_{a} \varphi_{+} ; & \eta_{b}=U_{b} \varphi_{+} / U_{b} \varphi_{-} ; \\
\theta_{a}=\left(U_{a} x / U_{a} \varphi_{+}\right)\left\|\varphi_{+}\right\|_{a} ; & \theta_{b}=\left(U_{b} x / U_{b} \varphi_{-}\right)\left\|\varphi_{-}\right\|^{b} ; \\
\theta_{a}^{*}=\eta_{a}\left\|\varphi_{+}\right\|_{a} ; & \theta_{b}^{*}=\eta_{b}\left\|\varphi_{-}\right\|^{b} ; \\
\omega_{-}<a \leqq a_{0}, & b_{0} \leqq b<\omega_{+} .
\end{aligned}
$$

The assumptions below turn out to be sufficient for $\mu_{a b}-\lambda$ and $\left\|y_{a b}-x\right\|_{a}^{b}$ to be $o(1)$ as $[a, b] \rightarrow\left(\omega_{-}, \omega_{+}\right)$.

Assumptions. ( $\omega_{-}$and $\omega_{+}$limit point singularities)

(i) The singularities $\omega_{-}$and $\omega_{+}$are not accumulation points of the zeros of $\varphi_{ \pm}$and

$$
\begin{aligned}
& \xi_{a}=o(1) \quad \text { and } \quad \xi_{a}^{*}=o(1) \quad \text { as } a \rightarrow \omega_{-} ; \\
& \xi_{b}=o(1) \quad \text { and } \quad \xi_{b}^{*}=o(1) \quad \text { as } b \rightarrow \omega_{+} .
\end{aligned}
$$

(ii) The boundary operators $U_{a}, U_{b}$ are restricted only by the boundedness of the quantities

1 The abbreviations $\|\varphi\|_{a},\|\varphi\|^{b}$ are used for $\|\varphi\|_{a}^{\omega_{+}},\|\varphi\|_{\omega-}^{b}$, following the convention of $\& 1$. 


$$
\begin{array}{ll}
\varphi_{+}(a) U_{a} \varphi_{-} / \varphi_{-}(a) U_{a} \varphi_{+} ; & \varphi_{+}(a) U_{a} x / x(a) U_{a} \varphi_{+} ; \\
\varphi_{-}(b) U_{b} \varphi_{+} / \varphi_{+}(b) U_{b} \varphi_{-} ; & \varphi_{-}(b) U_{b} x / x(b) U_{b} \varphi_{-}
\end{array}
$$

in some neighborhoods $\omega_{-}<a \leqq a_{0}, b_{0} \leqq b<\omega_{+}$of $\omega_{-}, \omega_{+}$respectively.

According to (6.3)-(6.8), these assumptions imply

$$
\sigma_{s}=o(1), \eta_{s}=o(1), \theta_{s}=o(1), \theta_{s}^{*}=o(1) \quad \text { as } s \rightarrow \omega_{ \pm} .
$$

The weaker assumptions $\theta_{s}=o(1), \theta_{s}^{*}=o(1)$ in (6.12) are actually sufficient for Theorem 4, while the stronger assumptions (6.9)-(6.11) are needed for the uniform estimate of Theorem 5 .

It follows from (6.3), (6.6), (6.11), and (6.12) that there exist constants $a_{0}, b_{0}$, and $C$ such that

$$
\left|\sigma_{a}\right| \leqq 1,\left|\sigma_{b}\right| \leqq 1,\left|\eta_{a}\right| \leqq C\left|\sigma_{a}\right|,\left|\eta_{b}\right| \leqq C\left|\sigma_{b}\right|
$$

provided $\omega_{-}<a \leqq a_{0}, b_{0} \leqq b<\omega_{+}$, and

$$
\begin{cases}\left|\sigma_{a}\right| \leqq\left|\sigma_{s}\right| & \text { if } \omega_{-}<a \leqq s \leqq a_{0} \\ \left|\sigma_{b}\right| \leqq\left|\sigma_{s}\right| & \text { if } b_{0} \leqq s \leqq b<\omega_{+}\end{cases}
$$

Condition (ii) above (6.11) is only a slight restriction on the boundary operators $U_{a}, U_{b}$. Compare (2.4) and (5.2) for limit circle problems of class 1 and 2 respectively. Sufficient conditions for the validity of (ii) when $\omega_{-}$is a regular singularity or an irregular singularity of finite rank are stated in [5, p. 840, p. 844]. In particular when $\omega_{-}=0$ is a regular singularity of $L_{0}$ with real, distinct exponents, then a sufficient condition for (ii) is that $\lim \left[-\alpha \alpha_{0}(\alpha) / \alpha_{1}(a)\right](\alpha \rightarrow 0)$ exist (finite or $\infty$ ) and be different from the smaller exponent.

We shall now describe a basic problem of the mixed type. It is enough to consider the case that $\omega_{-}$is a limit circle singularity and $\omega_{+}$is a limit point singularity. Then there exist solutions $\varphi_{ \pm}$of $L_{0} \varphi=0$ which satisfy

$$
\varphi_{+} \in \mathfrak{S}_{\mathcal{C}}, \varphi_{-} \in \mathfrak{F}_{\omega_{-} \omega},\left[\varphi_{-} \varphi_{-}\right](-)=0,\left[\varphi_{+} \bar{\varphi}_{-}\right](s)=1,
$$

where $\omega_{-}<\omega<\omega_{+}$, and these solutions will be determined once and for all by the fixed (but arbitrary) normalization $\left[\varphi_{+} \varphi_{+}\right]\left(s_{0}\right)=i$ $\left(\omega_{-}<s_{0}<\omega_{+}\right)$. Thus $\varphi_{+}$is described by (6.1) and $\varphi_{-}$is described by (2.1) in the mixed case.

Let $\mathfrak{D}^{0}$ be the basic domain described above (6.2) and let $\mathfrak{D}^{1}$ be the set of all $x \in \mathfrak{D}^{0}$ which satisfy the end condition $\left[x \varphi_{-}\right](-)=0$. The basic characteristic value problem in the mixed case is then

$$
L x=\lambda x, \quad x \in \mathfrak{D}^{1} .
$$

In the mixed case, assumptions (6.10) and the second of (6.11) are in 
effect at $\omega_{+}$together with the first assumption (2.4) at $\omega_{-}$.

Asymptotic estimates for the difference $\mu_{a b}-\lambda$ between characteristic values of (2.5) and (6.16) when $a, b$ are near $\omega_{-}, \omega_{+}$will be obtained in $\S 9$. The limit circle case has already been treated in $\S \S 3,4$ and the limit point case, when (6.2) replaces (6.16), will be treated in $\S 7$. Also uniform estimates for the difference $y_{a b}(s)-x(s)$ on $a \leqq$ $s \leqq b$ will be obtained under slightly stronger assumptions in $\S \S 8$ and 10. From these results, asymptotic variational formulae for characteristic values will be derived in $\S 11$.

7. Asymptotic estimates in the limit point case at both endpoints. When both $\omega_{-}$and $\omega_{+}$are limit point singularities, the basic problem is (6.2) and (2.5) is regarded as a perturbation of (6.2) arising from adjoining the boundary conditions $U_{a} y=U_{b} y=0$ at $s=a$ and $s=b$. The assumptions (6.9)-(6.11) are used in this section.

Let $G_{a b}(s, t)$ denote the Green's function for the differential operator $k L_{0}$ associated with the boundary conditions $U_{a} y=U_{b} y=0$, and let $G_{a b}$ denote the linear integral operator on $\mathfrak{F}_{a b}$ defined by the equation

$$
G_{a b} v(s)=\int_{a}^{b} G_{a b}(s, t) v(t) k(t) d t, v \in \widetilde{F}_{a b} .
$$

It is well-known [4, p. 20] that for any piecewise continuous function $v$ on $a \leqq s \leqq b$, the function $w=G_{a b} v$ is the unique solution in $\mathfrak{D}_{a b}$ [see (2.5)] of the differential equation $L_{0} w=v$.

Let $\lambda$ be a characteristic value of the basic problem (6.2) and let $x$ be a corresponding normalized characteristic function satisfying (6.9)(6.11). Define a function $f$ on $[a, b]$ by the equation ${ }^{2}$

$$
f=x-\gamma G_{a b} x, \text { where } \gamma=\lambda-l_{0} .
$$

Then $f$ is the unique solution of the boundary value problem $L_{0} f=0$, $U_{a} f=U_{a} x, U_{b} f=U_{b} x$, which has the following representation in terms of the functions $\varphi_{-}, \varphi_{+}$described by (6.1):

$$
\begin{aligned}
f(s) & =\left(\frac{U_{a} x}{U_{a} \varphi_{+}}\right)\left(\frac{\eta_{b} \varphi_{-}(s)-\varphi_{+}(s)}{\eta_{a} \eta_{b}-1}\right) \\
& +\left(\frac{U_{b} x}{U_{b} \varphi_{-}}\right)\left(\frac{\eta_{a} \varphi_{+}(s)-\varphi_{-}(s)}{\eta_{a} \eta_{b}-1}\right) .
\end{aligned}
$$

It follows from (6.7), (6.8) that

$\|f\|_{a}^{b} \leqq\left|1-\eta_{a} \eta_{b}\right|^{-1}\left(\left|U_{a} x\right| U_{a} \varphi_{+}|| \theta_{b}^{*}|+| \theta_{a}|+| U_{b} x\left|U_{b} \varphi_{-}\right|\left|\theta_{a}^{*}\right|+\left|\theta_{b}\right|\right)$

${ }^{2}$ The function on $[a, b]$ which coincides with $x$ on this interval will also be denoted. by $x$. 
According to (6.12), $\eta_{a}=o(1), \theta_{a}=o(1), \theta_{a}^{*}=o(1)$ as $a \rightarrow \omega_{-}$and $\eta_{b}=$ $o(1), \theta_{b}=o(1), \theta_{b}^{*}=o(1)$ as $b \rightarrow \omega_{+}$. Hence there exists a rectangle $R_{0}$ and a constant ${ }^{3} C$ on $R_{0}$ such that $\left|\eta_{a} \eta_{b}\right| \leqq \frac{1}{2}$ for $[a, b] \in R_{0}$, and

$$
\|f\|_{a}^{b} \leqq C\left(\left|\theta_{a}\right|+\left|\theta_{b}\right|\right) \text { for }[a, b] \in R_{0} .
$$

It follows from (7.2) and (7.4) that for any characteristic function $x$ associated with the characteristic value $\lambda$,

$$
\left\|x-\gamma G_{a b} x\right\|_{a}^{b} \leqq \mathrm{C}\left(\left|\theta_{a}\right|+\left|\theta_{b}\right|\right)\|x\| .
$$

Let $P(\delta)(\delta>0)$ be the projection from $\widetilde{F}_{a b}$ onto the subspace $\mathfrak{F}_{a b}(\delta)$ spanned by all characteristic functions $y^{i}$ of (2.5) whose corresponding $\mu^{i}$ lie in the interval $\left|\kappa^{i}-\lambda\right| \leqq \delta$. Then according to the fundamental lemma of $\S 2$,

$$
\|x-P(\delta) x\|_{a}^{b} \leqq(1+|\gamma| \mid \delta)\left\|x-\gamma G_{a b} x\right\|_{a}^{b} .
$$

The proof appears in [1]. With the aid of (7.5), we see that there exists a constant $C$ on $R_{0}$ such that

$$
\|x-P(\delta) x\|_{a}^{b} \leqq(C / 2 \delta)\left(\left|\theta_{a}\right|+\left|\theta_{b}\right|\right)\|x\|_{a}^{b}
$$

provided $[a, b] \in R_{0}$. With the choice $\delta=C\left(\left|\theta_{a}\right|+\left|\theta_{b}\right|\right)$ we conclude that $P\left(C\left|\theta_{a}\right|+C\left|\theta_{b}\right|\right) x=0$ implies that $x=0$ on $[a, b]$. Hence there exists at least one characteristic value $\mu=\mu_{a b}$ of (2.5) such that $\left|\mu_{a b}-\lambda\right| \leqq C\left(\left|\theta_{a}\right|+\left|\theta_{b}\right|\right)$ if $[a, b] \in R_{0}$. The proof that there is exactly one follows that in the limit circle case and will be omitted. $[6, \S 3]$ The following analogue of Theorem 3 is therefore valid:

THEOREM 4. If both singularities $\omega_{-}$and $\omega_{+}$of the differential operator $L$ are of the limit point type, under the assumptions (6.9)(6.11), (or even under the weaker assumptions $\theta_{s}=o(1), \theta_{s}^{*}=o(1)$ as $\left.s \rightarrow \omega_{ \pm}\right)$then for every basic characteristic value $\lambda$ of (6.2) there exists a rectangle $R_{0}$ and a constant $C$ on $R_{0}$ such that a unique $\mu_{a b}$ satisfies $\left|\mu_{a b}-\lambda\right| \leqq C\left(\left|\theta_{a}\right|+\left|\theta_{b}\right|\right)$ whenever $[a, b] \in R_{0}$. There are normalized characteristic functions $x, y_{a b}$ associated with $\lambda, \mu_{a b}$ respectively such that $\left\|y_{a b}-x\right\|_{a}^{b} \leqq C\left(\left|\theta_{a}\right|+\left|\theta_{b}\right|\right)$.

8. Uniform estimates in the limit point case. In order to obtain uniform estimates for $y_{a b}(s)-x(s)$ on $a \leqq s \leqq b$, following the method of $\S 4$, we need stronger assumptions than (6.9)-(6.11). It will be supposed in addition that the following are bounded on $\omega_{-}<s<\omega_{+}$;

$$
\varphi_{+}(s)\left\|\varphi^{-}\right\|^{s} ; \quad \varphi_{-}(s)\left\|\varphi_{+}\right\|_{s}
$$

\footnotetext{
${ }^{3} C$ will be used throughout as a generic notation for a constant on $R_{0}$.
} 
Let $a_{0}, b_{0}$ be the fixed numbers in (6.11)-(6.14) and let $\hat{\varphi}_{ \pm}(s)$ be defined by

$$
\begin{aligned}
\hat{\varphi}_{ \pm}(s) & =\left|\varphi_{ \pm}(s)\right| & & \text { if } \omega_{-}<s<a_{0}, b_{0}<s<\omega_{+} \\
& =1 & & \text { if } a_{0} \leqq s \leqq b_{0} .
\end{aligned}
$$

We assert that there exists a constant $C$, independent of $a, b$ as well as $s$, such that

$$
\begin{aligned}
& \left|\eta_{a} \varphi_{+}(s)\right| \leqq C \hat{\varphi}_{-}(s) \quad \text { on } a \leqq s \leqq b, a \leqq a_{0} \\
& \left|\eta_{b} \varphi_{-}(s)\right| \leqq C \hat{\varphi}_{+}(s) \quad \text { on } a \leqq s \leqq b, b_{0} \leqq b .
\end{aligned}
$$

These inequalities are obvious on the fixed intervals $a_{0} \leqq s \leqq b_{0}$. To complete the proof of (8.4), we deduce from (6.3), (6.13), and (6.14) that

$$
\left|\eta_{b} \varphi_{-}(s)\right| \leqq C\left|\sigma_{b} \varphi_{-}(s)\right| \leqq C\left|\sigma_{s} \varphi_{-}(s)\right|=C\left|\varphi_{+}(s)\right|
$$

on $b_{0} \leqq s \leqq b<\omega_{+}$. Since $\left|\sigma_{s}\right|=\left|\varphi_{-}(s) / \varphi_{+}(s)\right| \leqq 1$ on $\omega_{-}<s \leqq a_{0}$, by (6.13), it follows that $\left|\eta_{b} \varphi_{-}(s)\right| \leqq C\left|\varphi_{+}(s)\right|$ on $\omega_{-}<a \leqq s \leqq a_{0}$ as well. Thus (8.4) is valid on the whole interval $a \leqq s \leqq b$. The proof of (8.3) is similar and will be omitted.

The Green's function $G_{a b}(s, t)$ for $L$ on $\mathfrak{D}_{a b}$ (associated with the boundary conditions $U_{a} y=U_{b} y=0$ ) is given by

$$
\begin{aligned}
G_{a b}(s, t) & =\Omega^{-1} \psi_{a}(t) \psi_{b}(s) \text { if } a \leqq t \leqq s \leqq b, \\
& =\Omega^{-1} \psi_{a}(s) \psi_{b}(t) \quad \text { if } a \leqq s \leqq t \leqq b,
\end{aligned}
$$

where

$$
\begin{aligned}
\psi_{a}(s) & =\varphi_{-}(s) U_{a} \varphi_{+}-\varphi_{+}(s) U_{a} \varphi_{-}, \\
\psi_{b}(s) & =\varphi_{-}(s) U_{b} \varphi_{+}-\varphi_{+}(s) U_{b} \varphi_{-}, \\
\Omega & =U_{a} \varphi_{-} U_{b} \varphi_{+}-U_{a} \varphi_{+} U_{b} \varphi_{-} .
\end{aligned}
$$

Let $G_{a b}$ denote the Green's operator (7.1). It will first be shown that $\gamma G_{a b} x(s)$ is uniformly close to $y(s)$ on $a \leqq s \leqq b$ when $a, b$ are near $\omega_{-}, \omega_{+}$. The following lemma will be needed in the proof.

Lemma 2. The positive function $g_{a b}$ defined by

$$
g_{a b}^{2}(s)=\int_{a}^{b}\left|G_{a b}(s, t)\right|^{2} k(t) d t
$$

is uniformly bounded on $a \leqq s \leqq b$ provided $a \leqq a_{0}, b_{0} \leqq b$.

Proof. According to (6.6), (8.5), and (8.6), $g_{a b}(s)$ has the following explicit representation 


$$
\begin{aligned}
g_{a b}^{2}(s)= & \left|1-\eta_{a} \eta_{b}\right|^{-2}\left[\left(\left|\eta_{b} \varphi_{-}(s)-\varphi_{+}(s)\right||| \varphi_{-}-\eta_{a} \varphi_{+} \|_{a}^{s}\right)^{2}\right. \\
& \left.+\left(\left|\varphi_{-}(s)-\eta_{a} \varphi_{+}(s)\right||| \eta_{b} \varphi_{-}-\varphi_{+} \|_{s}^{b}\right)^{2}\right] .
\end{aligned}
$$

It then follows from (8.3), (8.4) that there exists a constant $C$ such that

$$
g_{a b}^{2}(s) \leqq\left|1-\eta_{a} \eta_{b}\right|^{-2} C\left[\left(\left.\widehat{\varphi}_{+}(s)\left\|\hat{\varphi}_{-}\right\|\right|_{a} ^{s}\right)^{2}+\left(\hat{\varphi}_{-}(s)\left\|\hat{\varphi}^{+}\right\|_{s}^{b}\right)^{2}\right]
$$

Since $\left|\eta_{a} \eta_{b}\right| \leqq \frac{1}{2}$ on $\omega_{-}<a \leqq a_{0}, b_{0} \leqq b<\omega_{+}$, the conclusion of Lemma 2 is therefore a consequence of the hypothesis (8.1).

The Schwarz inequality for $\mathfrak{F}_{a b}$ yields

$$
\begin{gathered}
\left|y_{a b}(s)-\left(\lambda-l_{0}\right) G_{a b} x(s)\right|=\left|G_{a b}\left[\left(\mu_{a b}-l_{0}\right) y_{a b}(s)-\left(\lambda-l_{0}\right) x(s)\right]\right| \\
\leqq g_{a b}(s)\left(\left|\mu_{a b}-l_{0}\right||| y_{a b}-x\left\|_{a}^{b}+\left|\mu_{a b}-\lambda\right|\right\| x \|\right) .
\end{gathered}
$$

Hence Lemma 2 and Theorem 4 show that there exists $C$ such that

$$
\left|y_{a b}(s)-\left(\lambda-l_{0}\right) G_{a b} x(s)\right| \leqq C\left(\left|\theta_{a}\right|+\left|\theta_{b}\right|\right),
$$

$a \leqq s \leqq b$ whenever $a \leqq a_{0}, b_{0} \leqq b$.

The solution $f(s)$ of the boundary value problem $L_{0} f=0, U_{a} f=$ $U_{a} x, U_{b} f=U_{b} x$ is given by (7.2) or (7.3). The function $F$ defined by

$$
F(s)=\left(\lambda-l_{0}\right) G_{a b} x(s)-x(s)+f(s)
$$

satisfies $L_{0} F=0, U_{a} F=U_{b} F=0$, and hence $F$ is the zero function on $a \leqq s \leqq b$. The following uniform estimate is then an immediate consequence of (8.8):

$$
\begin{aligned}
y_{a b}(s) & =x(s)-f(s)+O\left(\theta_{a}\right)+O\left(\theta_{b}\right), \\
a & \leqq s \leqq b, \omega_{-}<a \leqq a_{0}, b_{0} \leqq b<\omega_{+} .
\end{aligned}
$$

Theorem 5. If both singularities $\omega_{-}$and $\omega_{+}$of $L$ are of the limit point type, under the assumptions (6.9)-(6.11), (8.1), the perturbed characteristic function $y_{a b}$ associated with the characteristic value $\mu_{a b}$ of Theorem 4 has the uniform representation (8.9).

9. Asymptotic estimates in the mixed case. In this section, $\omega_{-}$ is supposed to be a limit circle singularity and $\omega_{+}$a limit point singularity. The basic problem is (6.16) and the assumptions are (6.10), the second of (6.11), and the first of (2.4).

Proceeding as in $\S 7$, we obtain the representation (7.3) and the inequality below (7.3) where $\varphi_{ \pm}$are described by (6.15) in the mixed case. According to (6.12), the following relations hold in connection with the limit point singularity $\omega_{+}: \eta_{b}=o(1), \theta_{b}^{*}=o(1)$, and $U_{b} x / U_{b} \varphi_{-}=$ $O\left(\theta_{b}\right)=o(1)$ as $b \rightarrow \omega_{+}$. Since $\omega_{-}$is a limit circle singularity, it is a consequence of (3.6) that 


$$
\theta_{a}^{*}=\left(U_{a} \varphi_{-} / U_{a} \varphi_{+}\right)\left\|\varphi_{+}\right\|_{a}=o(1) ; \eta_{a}=o(1) \quad \text { as } a \rightarrow \omega_{-} .
$$

In addition to (6.3)-(6.8) we shall use the notation

$$
\rho_{a}=U_{a} x \text {. }
$$

It follows from the postulated end condition $\left[x \varphi_{-}\right](-)=0$ that for $x \in \mathfrak{D}^{1}, \rho_{a}=\left[x \varphi_{-}\right](a)[1+o(1)]=o(1)$ as $a \rightarrow \omega_{-}$, and from (3.6),

$$
\theta_{a}=\left(U_{a} x / U_{a} \varphi_{+}\right)\left\|\varphi_{+}\right\|_{a}=O\left(\rho_{a}\right), \omega_{-}<a \leqq a .
$$

The analogue of (7.4) in the mixed case is therefore

$$
\|f\|_{a}^{b} \leqq C\left(\left|\rho_{a}\right|+\left|\theta_{b}\right|\right) .
$$

The proof of the following theorem is then identical with that of Theorem 4.

THEOREM 6. If $\omega_{-}$is a limit circle singularity and $\omega_{+}$is a limit point singularity of $L$, then under the assumptions (6.10), (6.11), and the first of (2.4), for every $\lambda$ of the mixed problem (6.16) there exists $R_{0}$ and a constant $C$ on $R_{0}$ such that a unique $\mu_{a b}$ of (2.5) lies in the interval $\left|\mu_{a b}-\lambda\right| \leqq C\left(\left|\rho_{a}\right|+\left|\theta_{b}\right|\right)$ whenever $[a, b] \in R_{0}$. There are normalized characteristic functions $x, y_{a b}$ associated with $\lambda, \mu_{a b}$ respectively such that

$$
\left\|y_{a b}-x\right\| \leqq C\left(\left|\rho_{a}\right|+\left|\theta_{b}\right|\right) .
$$

10. Uniform estimates in the mixed case. To obtain uniform estimates for characteristic functions on $a \leqq s \leqq b$ in the mixed case, we assume instead of (8.1) that the following are bounded

$$
\varphi_{ \pm}(s) \text { on } \omega_{-}<a \leqq a_{0} ; \quad \varphi_{+}(s)\left\|\varphi_{-}\right\|^{s} \text { on } \omega_{-}<s<\omega_{+} \cdot
$$

Equation (8.7) holds also in the mixed case, and $\eta_{a}=o(1)$ as $a \rightarrow \omega_{-}$ by (9.1) as well as $\eta_{b}=o(1)$ as $b \rightarrow \omega_{+}$. Since $\left\|\varphi_{+}\right\|$exists by (6.15), there exists a constant $C$ such that

$$
\begin{aligned}
& \left\|\varphi_{-}-\eta_{a} \varphi_{+}\right\|_{a}^{s} \leqq C\left\|\varphi_{-}\right\|^{s}, \\
& \left\|\eta_{b} \varphi_{-}-\varphi_{+}\right\|_{s}^{b} \leqq C\left|\eta_{b}\right|\left\|\varphi_{-}\right\|^{b}=C \theta_{b}^{*}
\end{aligned}
$$

and $\theta_{b}^{*}=o(1)$ as $b \rightarrow \omega_{+}$. Since $\varphi_{ \pm}(s)$ are bounded on $\omega_{-}<s \leqq b_{0}$ by (10.1), $g_{a b}(s)$ is bounded on $a \leqq s \leqq b_{0}$. To show that $g_{a b}(s)$ is bounded also on $b_{0}<s \leqq b<\omega_{+}$, we obtain as in the proof of (8.3), (8.4) that

$$
\left|\eta_{a} \varphi_{+}(s)\right| \leqq C\left|\varphi_{-}(s)\right|, \quad\left|\eta_{b} \varphi_{-}(s)\right| \leqq C\left|\varphi_{+}(s)\right|
$$

and hence by (8.7), (10.2), 


$$
\begin{aligned}
g_{a b}^{2}(s) & \leqq\left|1-\eta_{a} \eta_{b}\right|^{-2} C_{1}\left[\left(\left|\varphi_{+}(s)\right||| \varphi_{-} \|^{s}\right)^{2}+\left(\left|\eta_{b} \varphi_{-}(s)\right||| \varphi_{-} \|^{b}\right)^{2}\right] \\
& \leqq\left|1-\eta_{a} \eta_{b}\right|^{-2} C_{2}\left(\left|\varphi_{+}(s)\right|\left\|\varphi_{-}\right\|^{s}\right)
\end{aligned}
$$

for some constants $C_{1}, C_{2}$. Then $g_{a b}(s)$ is bounded by the hypothesis (10.1). The following analogue of Theorem 5 is then valid.

THEOREM 7. If $\omega_{-}$is a limit circle singularity and $\omega_{+}$is a limit point singularity of $L$, then under the assumptions (6.10), the second of (6.11), the first of (2.4), and (10.1), the characteristic function $y_{a b}$ associated with the characteristic value $\mu_{a b}$ of Theorem 6 has the following uniform asymptotic representation:

$$
\begin{aligned}
& y_{a b}(s)=x(s)-f(s)+O\left(\rho_{a}\right)+O\left(\theta_{h}\right) \\
& a \leqq s \leqq b, \quad \omega_{-}<a \leqq a_{0}, \quad b_{0} \leqq b<\omega_{+}
\end{aligned}
$$

where $f$ is given by (7.2).

11. Asymptotic variational formulae for characteristic values. The purpose here is to derive formulae for the change $\mu_{a b}-\lambda$ of characteristic values under the perturbation $\mathfrak{D}^{0} \rightarrow \mathfrak{D}_{a b}$, valid for $a, b$ in neighborhoods of $\omega_{-}, \omega_{+}$respectively.

Let $x, y$ denote the normalized characteristic functions associated with $\lambda, \mu$ as described in Theorems 4 and 5 . Let $f$ be the solution (7.3) of the boundary value problem

$$
L_{0} f=0, \quad U_{a} f=U_{a} x, \quad U_{b} f=U_{b} x .
$$

We conclude from the boundary conditions $U_{a} y=U_{b} y=0$ that $[x y](a)=$ $[f y](a)$ and $[x y](b)=[f y](b)$. Then application of Green's formula

$$
(L x, y)_{a}^{b}-(x, L y)_{a}^{b}=[x y](b)-[x y](a)
$$

to the differential equations $L x=\lambda x, L y=\mu y$, and $L f=l_{0} f$ on $[a, b]$ leads to

$$
\begin{aligned}
(\lambda-\mu)(x, y)_{a}^{b} & =\left(l_{0}-\mu\right)(f, y)_{a}^{b} ; \\
{[f x](b)-[f x](a) } & =\left(l_{0}-\lambda\right)(f, x)_{a}^{b} .
\end{aligned}
$$

We obtain as a consequence of Theorem 4 that $\mu=\lambda+o(1)$ and

$$
\left|(x, y)_{a}^{b}-(x, x)_{a}^{b}\right| \leqq\|x\|\|y-x\|_{a}^{b}=o(1)
$$

as $a, b \rightarrow \omega_{-}, \omega_{+}$. Hence

$$
(x, y)_{a}^{b}=1+o(1), \quad a, b \rightarrow \omega_{-}, \omega_{+}
$$

and (11.1) yields 


$$
\lambda-\mu=\left(l_{0}-\lambda\right)(f, y)_{a}^{b}[1+o(1)] .
$$

We now appeal to the uniform estimate (8.9) to obtain

$$
(f, y)_{a}^{b}=(f, x)_{a}^{b}-(f, f)_{a}^{b}+\left(\theta_{a}+\theta_{b}\right)(f, 1)_{a}^{b} \bigcirc(1) .
$$

The following asymptotic variational formula is then a consequence of (11.2) and (11.3):

$$
\lambda-\mu_{a b}=[f x](b)-[f x](a)+\left(l_{0}-\lambda\right)(f, f)_{a}^{b}+\left(\theta_{a}+\theta_{b}\right)(f, 1)_{a}^{b} O(1) .
$$

In various problems of practical interest (see [5], [6] for detailed references) the first two terms on the right dominate the other terms, and the asymptotic relation

$$
\lambda-\mu_{a b} \sim[f x](b)-[f x](a)
$$

is valid for $a, b \rightarrow \omega_{-}, \omega_{+}$. In some cases, $\lambda=0$ is not a characteristic value and it is permissible to replace $l_{0}$ by 0 . Then $f$ can be taken as a real valued solution of $L f=0$.

ExAmple 1. The Hermite operator $L$ given by $L x=-x^{\prime \prime}+s^{2} x$ will be considered on the interval $-\infty<s<\infty$. In this example, $k(s)=p(s)=1, q(s)=s^{2}, \omega_{-}=-\infty$, and $\omega_{+}=\infty$. Both singularities are limit point, and the basic problem (6.1) has characteristic values $\lambda^{(n)}=2 n+1$ and normalized characteristic functions

$$
x_{n}(s)=\pi^{-1 / 4} 2^{-(n+1) / 2}(n !)^{-1} \exp \left(-s^{2} / 2\right) H_{n}(s), \quad n=0,1, \cdots
$$

where $H_{n}(s)$ denotes an Hermite polynomial. The well-known [3] asymptotic behavior of $x_{n}(s)$ as $s \rightarrow \infty$ is

$$
x_{n}(s) \sim \pi^{-1 / 4} 2^{(n+1) / 2}(n !)^{-1 / 2} s^{n} \exp \left(-s^{2} / 2\right) .
$$

The perturbed problem to be considered is $L y=\mu y, y(a)=y(b)=0$. In this case $l_{0}$ can be replaced by 0 , and the solutions $\varphi_{+}$and $\varphi_{-}$of $L \varphi=0$ have the asymptotic behavior

$$
\log \varphi_{ \pm}(s) \sim \pm \frac{1}{2} s^{2}
$$

as $s \rightarrow-\infty$;

We then obtain from the representation (7.3) of $f(s)$ that $f^{\prime}(a) \sim a x(a)$ as $a \rightarrow-\infty$. Since $x^{\prime}(a) \sim-x(a)$, $[x f](a) \sim 2 a x^{2}(a)$. Similarly $[x f](b) \sim$ $2 b x^{2}(b)$. Then (11.4), (11.5) give the asymptotic variational formula

$$
\begin{array}{cc}
\mu_{a b}^{(n)} \sim 2 n+1+\pi^{-1 / 2} 2^{n+2}(n !)^{-1}\left[b^{2 n+1} \exp \left(-b^{2}\right)-a^{2 n+1} \exp \left(-a^{2}\right)\right] \\
a, b \rightarrow-\infty, \infty ; & n=0,1,2, \cdots .
\end{array}
$$

ExAmple 2. Consider the confluent hypergeometric operator $L$ 
given by

$$
L x=s\left[-\frac{d^{2} x}{d s^{2}}+\frac{x}{4}+\frac{j(j+1)}{s^{2}} x\right], \quad 0<s<\infty
$$

in which $j$ is a nonnegative integer. This is related to the Laguerre differential equation, which arises in he quantum mechanical theory of the Hydrogen atom [3]. In this example, $k(s)=1 / s, p(s)=1$, and $q(s)=j(j+1) s^{-2}+1 / 4$. The singularity $\omega_{+}=\infty$ is in the limit point case, and $\omega_{-}=0$ is in the limit point or limit circle case according as $j \geqq 1$ or $j=0$. If $j=0$, the singularity is a class 1 limit circle singularity $(\$ 5)$ and it can be verified that the variational formula (11.4) is still valid. The basic problem (6.2) has characteristic values $\lambda^{(n)}=n(n \geqq j+1=1,2, \cdots)$ and normalized characteristic functions [3]

$$
x_{n j}(s)=-[(n-j-1) !]^{1 / 2}[(n+j) !]^{-3 / 2} s^{j+1} e^{-s / 2} L_{n+j}^{2 j+1}(s),
$$

where $L_{\imath}^{h}(s)$ denotes the associated Laguerre polynomial, with the asymptotic behaviour

$$
\begin{array}{ll}
x_{n j}(s) \sim(-1)^{n-j-1}[(n+j) !]^{-1 / 2}[(n-j-1) !]^{-1 / 2} s^{n} e^{-s / 2}, & s \rightarrow \infty \\
x_{n j}(s) \sim[(n+j) !]^{1 / 2}[(n-j-1) !]^{-1 / 2}[(2 j+1) !]^{-1} s^{j+1}, & s \rightarrow 0 .
\end{array}
$$

The normal solutions of $L \varphi=0$ have the asymptotic behaviour

$$
\log \varphi_{ \pm}(s) \sim \mp \frac{1}{2} s \pm n \log s \quad(s \rightarrow \infty) .
$$

For a perturbed problem with boundary operators $U_{a} x=x(a)$, $U_{b} x=x(b)$, the representation $(7.3)$ gives $f^{\prime}(b) \sim x(b) \varphi_{-}^{\prime}(b) / \varphi_{-}(b)$, or $f^{\prime}(b) \sim \frac{1}{2} x(b)$ as $b \rightarrow \infty$. Similarly $f^{\prime}(a) \sim-j x(a) / a$ as $a \rightarrow 0$. Hence

$$
[x f](a) \sim-(2 j+1) a^{-1} x^{2}(a) ; \quad[x f](b) \sim x^{2}(b),
$$

and (11.4), (11.6), (11.7) yield the asymptotic formula

$$
\begin{gathered}
\mu_{a b}^{(n)} \sim n+\frac{(2 j+1)(n+j) ! a^{2 j+1}}{(n-j-1) ![(2 j+1) !]^{2}}+\frac{b^{2 n} e^{-b}}{(n+j) !(n-j-1) !} \\
a \rightarrow 0, \quad b \rightarrow \infty, \quad j+1 \leqq n=1,2, \cdots .
\end{gathered}
$$

To solve the perturbed problem

$$
\frac{d^{2} y}{d S^{2}}+\left[\frac{2}{S}-\frac{j(j+1)}{S^{2}}+\nu\right] y=0, \quad y(A)=y(B)=0,
$$

we transform the differential equation into the form $L y=\mu y$ of example 2 by the change of variables 


$$
S=\mu s / 2, A=\mu a / 2, B=\mu b / 2, \nu=-1 / \mu^{2}
$$

and obtain the result

$$
\nu_{A B}^{(n)}+\frac{1}{n^{2}} \sim \frac{2}{n^{3}}\left(\mu_{a b}^{(n)}-n\right) \quad(A \rightarrow 0 ; B \rightarrow \infty) .
$$

\section{REFERENCES}

1. H. F. Bohnenblust, C. R. DePrima, and C. A. Swanson, Elliptic operators with perturbed domain, To be published.

2. E. A. Coddington and N. Levinson, Theory of ordinary differential equations, McGraw-Hill (1955).

3. P. M. Morse and H. Feshbach, Methods of theoretical physics, McGraw-Hill (1953).

4. F. Rellich, Spectral theory of a second order ordinary differential operator, New York University (1953).

5. C. A. Swanson, Differential operators with perturbed domains, J. of rational mechanics and analysis, 6, 6 (1957) 823-846.

6. _- Asymptotic estimates for limit circle problems, Pacific J. Math., 11 (1961), 1549-1559.

The University of British Columbia 


\section{PACIFIC JOURNAL OF MATHEMATICS}

\section{EDITORS}

\section{RalPh S. Phillips}

Stanford University

Stanford, California

M. G. Arsove

University of Washington

Seattle 5, Washington
J. Dugundji

University of Southern California Los Angeles 7, California

Lowell J. Paige

University of California

Los Angeles 24, California

\section{ASSOCIATE EDITORS}
E. F. BECKENBACH
D. DERRY
H. L. ROYDEN
E. G. STRAUS
T. M. CHERRY
M. OHTSUKA
E. SPANIER
F. WOLF

\section{SUPPORTING INSTITUTIONS}

\author{
UNIVERSITY OF BRITISH COLUMBIA \\ CALIFORNIA INSTITUTE OF TECHNOLOGY \\ UNIVERSITY OF CALIFORNIA \\ MONTANA STATE UNIVERSITY \\ UNIVERSITY OF NEVADA \\ NEW MEXICO STATE UNIVERSITY \\ OREGON STATE UNIVERSITY \\ UNIVERSITY OF OREGON \\ OSAKA UNIVERSITY \\ UNIVERSITY OF SOUTHERN CALIFORNIA
}

STANFORD UNIVERSITY

UNIVERSITY OF TOKYO

UNIVERSITY OF UTAH

WASHINGTON STATE UNIVERSITY

UNIVERSITY OF WASHINGTON

AMERICAN MATHEMATICAL SOCIETY

CALIFORNIA RESEARCH CORPORATION SPACE TECHNOLOGY LABORATORIES

NAVAL ORDNANCE TEST STATION

Printed in Japan by International Academic Printing Co., Ltd., Tokyo Japan 


\section{Pacific Journal of Mathematics}

\section{Vol. 13, No. 1 \\ March, 1963}

Frantz Woodrow Ashley, Jr., A cone of super-(L) functions............. 1

Earl Robert Berkson, Some metrics on the subspaces of a Banach space....

Felix Earl Browder and Walter Strauss, Scattering for non-linear wave

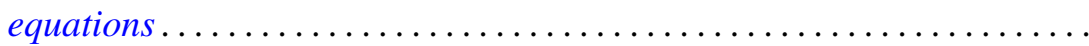

Edmond Darrell Cashwell and C. J. Everett, Formal power series ..........

Frank Sydney Cater, Continuous linear functionals on certain topological

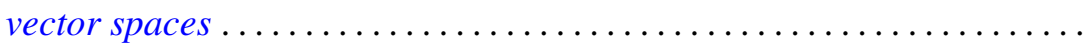

John Douglas Dixon, General group extensions ....................

Robert Pertsch Gilbert, On harmonic functions of four variables with

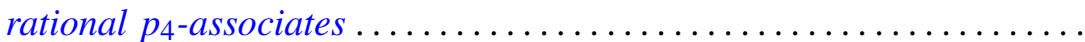

Irving Leonard Glicksberg, On convex hulls of translates ..............

Simon Hellerstein, On a class of meromorphic functions with deficient zeros

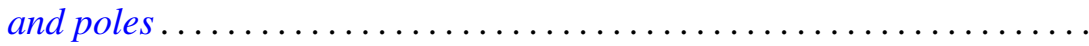

Donald William Kahn, Secondary cohomology operations which extend the

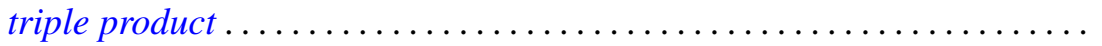

G. K. Leaf, A spectral theory for a class of linear operators .............

R. Sherman Lehman, Algebraic properties of the composition of solutions of partial differential equations ........................... 157

Joseph Lehner, On the generation of discontinuous groups ............. 169

S. P. Lloyd, On certain projections in spaces of continuous functions ...... 171 Fumi-Yuki Maeda, Generalized spectral operators on locally convex spaces ..................................

Donald Vern Meyer, $E^{3}$ modulo a 3-cell

William H. Mills, An application of linear programming to permutation groups.

Richard Scott Pierce, Centers of purity in abelian groups

Christian Pommerenke, On meromorphic starlike functions ...

Zalman Rubinstein, Analytic methods in the study of zeros of

polynomials...

B. N. Sahney, On the Nörlund summability of Fourier series

Tôru Saitô, Regular elements in an ordered semigroup . .

Lee Meyers Sonneborn, Level sets on spheres...........

Charles Andrew Swanson, Asymptotic estimates for limit point

problems .

Lucien Waelbroeck, On the analytic spectrum of Arens . .

Alvin (Murray) White, Singularities of a harmonic function of three

variables given by its series development .............

Kōichi Yamamoto, Decomposition fields of difference sets ...

Chung-Tao Yang, On the action of $\mathrm{SO}(3)$ on a cohomology manifold... 